\begin{tabular}{|c|c|c|c|}
\hline & & $\begin{array}{c}\text { ISSN = } 1980-993 X-\text { doi:10.4136/1980-993X } \\
\text { www.agro.unitau.br/ambi-agua } \\
\text { E-mail: ambi-agua@agro.unitau.br } \\
\text { Tel.: (12) 3625-4116 }\end{array}$ & \\
\hline
\end{tabular}

\title{
Variabilidade espacial de vazão e pressão em subunidade de microaspersão com emissores usados e novos
}

(doi:10.4136/ambi-agua.62)

\author{
Wagner Walker de Albuquerque Alves ${ }^{1}$; José Dantas Neto²; José de Arimatea de \\ Matos $^{3}$; Carlos Alberto Vieira de Azevedo²; Vera Lúcia Antunes de Lima ${ }^{2}$ \\ ${ }^{1}$ Bolsista Pós-Doutorado-CNPq, UFCG/CTRN, Departamento de Engenharia Agrícola \\ E-mail: wagnerwaa@gmail.com \\ ${ }^{2}$ Professores do Departamento de Engenharia agrícola da UFCG \\ E-mail: \{cazevedo, zedantas, antunes\}@deag.ufcg.edu.br \\ ${ }^{3}$ Professor do Departamento de Ciências Ambientais, UFERSA \\ E-mail: jamatos@ufersa.edu.br
}

\section{RESUMO}

Este trabalho estudou em campo a distribuição espacial de vazão e pressão em subunidade irrigada por microaspersão, considerando emissores usados e novos. Os dados foram coletados em 28 pontos de emissão espaçados em $10 \times 18 \mathrm{~m}$. Os valores médios foram submetidos a teste de normalidade e também a uma análise geoestatística; a verificação da dependência espacial e da interpolação foi realizada pelo método da krigagem e as continuidades espaciais foram estudadas, construindo-se semivariogramas para as vazões e pressões da subunidade com microaspersores novos e usados. Verifica-se que os modelos variaram se o emissor era novo ou usado. O modelo matemático que melhor se ajustou à vazão dos microaspersores novos e usados foi, respectivamente, o exponencial e esférico; para a pressão o modelo foi o inverso da vazão, ou seja, esférico para os novos e exponencial para os usados. Os valores para os emissores usados do efeito pepita $(\mathrm{Co})$, patamar $(\mathrm{Co}+\mathrm{Cl}) \mathrm{e}$ alcance (Ao) foram de $1,0 \mathrm{~L} \mathrm{~h}^{-1}, 33 \mathrm{~L} \mathrm{~h}^{-1}$ e $218 \mathrm{~m}$ e de $0,43,137 \mathrm{kPa}$ e $58 \mathrm{~m}$, para vazão e pressão, respectivamente. O coeficiente de uniformidade de irrigação, o coeficiente de uniformidade do sistema e a eficiência de aplicação d’água aumentaram, respectivamente, em $11,9,10,58$ e 10,75\%, quando os emissores usados foram substituídos por novos. A distância máxima (alcance) onde os dados estimados de vazão e pressão se correlacionam espacialmente extrapolou o comprimento da linha de derivação para a vazão com microaspersores novos e usados e para a pressão só com microaspersores novos. O efeito pepita para a pressão no sistema foi menor quando se trocou os microaspersores usados por novos, acontecendo o inverso para a vazão. Houve um aumento tanto da pressão como da vazão quando se trocou os microaspersores usados por novos.

Palavras-chave: microaspersor; geoestatística; avaliação.

\section{Spatial variability of discharge and pressure in subunit of microsprinkler irrigation with used and new emitters}

\begin{abstract}
This work studied in field the spatial distribution of discharge and pressure in subunit irrigated by microsprinkler, considering used and new emitters. The data were collected in 28 emission points spaced in $10 \times 18 \mathrm{~m}$. The mean values were submitted to normality test and
\end{abstract}


also to a geostatistical analysis; the verification of the spatial dependence and of the interpolation was accomplished by the method of krigagem and the spatial continuities were studied by means of semivariograms for the discharges and pressures of the subunit with new and used microsprinklers. It is verified that the models varied if the emitter was new or used. The mathematical model that better adjusted to the discharge of the new and used emitters was, respectively, exponential and spherical; for the pressure, the model was the inverse of the discharge, that is, spherical for new emitters and exponential for used ones. The values for the used emitters of the nugget effect $(\mathrm{Co})$, landing $(\mathrm{Co}+\mathrm{Cl})$ and reach $(\mathrm{Ao})$ were of $1.0 \mathrm{~L} \mathrm{~h}^{-1}$, $33 \mathrm{~L} \mathrm{~h}^{-1}$ and $218 \mathrm{~m}$ and of $0.43,137 \mathrm{kPa}$ and $58 \mathrm{~m}$, for discharge and pressure, respectively. The coefficient of irrigation uniformity, the coefficient of uniformity of the system and the water application efficiency increased, respectively, in 11.9, 10.58 and $10.75 \%$, when the used emitters were substituted by new ones. The maximum distance (reach) where the estimated data of discharge and pressure are spatially correlated extrapolated the length of the derivation line for the discharge with new and used microsprinklers and for the pressure only with new microsprinklers. The nugget effect for the pressure in the system was smaller when it was changed the used microasprinklers by new ones, the inverse was observed for the discharge. There was an increase in pressure as well as in discharge when the used microsprinklers were changed by new ones.

Keywords: microsprinkler; geostatistics; evaluation.

\section{INTRODUÇÃO}

Após a implantação de um projeto de irrigação, o produtor rural deve ser orientado para que possa obter o máximo de rendimento do sistema em uso. Isso implica não apenas estabelecer a adequação do momento de se efetuar as irrigações e da lâmina de água a ser aplicada, mas, também, uma uniformidade na aplicação da água desejável. Keller e Bliesner (1990) comentam que é recomendável, após a instalação de um sistema de irrigação, procederam-se testes de campo no intuito de se verificar a adequação da irrigação que foi projetada, para recomendar, caso haja necessidade, ajuste no manejo. Esses procedimentos visam maximizar a eficiência do sistema de irrigação.

De acordo com Keller e Karmeli (1974), apesar das inúmeras vantagens apresentadas, existem problemas na irrigação localizada, dentre os quais se destaca a obstrução dos emissores. Ela é causada por materiais orgânicos em suspensão, por deposição química e por partículas minerais. Essa obstrução afeta a uniformidade de aplicação d’água, que depende fundamentalmente das vazões dos emissores do sistema. À medida que se prolonga o tempo de uso dos equipamentos de um sistema de irrigação no decorrer do ciclo da cultura, aumenta a possibilidade de obstrução dos orifícios, diminuindo, conseqüentemente, a uniformidade com que a água é distribuída no campo, o que afeta o rendimento da cultura; necessita-se, então, de uma avaliação periódica da uniformidade de distribuição da água.

A uniformidade da irrigação tem efeito no rendimento das culturas, sendo considerada um dos fatores mais importantes no dimensionamento e na operação de sistemas de irrigação. Baixa uniformidade resulta em alto consumo de água e energia. As causas da desuniformidade de aplicação de água nos sistemas de irrigação localizados são as seguintes: diferenças de pressão que se produzem na rede devido às perdas de carga e à irregularidade da topografia do terreno; insatisfatória uniformidade de fabricação dos emissores, devido ao inadequado controle de qualidade; número de emissores por planta; variação das características hidráulicas do emissor, ao longo do tempo, devido a possíveis obstruções e/ou 
envelhecimento; temperatura da água; efeito do vento; e variação de fabricação dos reguladores de pressão, quando existirem (Abreu et al., 1987). A utilização de sistemas de irrigação mais eficientes é uma busca constante na agricultura irrigada, pois existe tendência de aumento no custo da energia e de redução da disponibilidade hídrica dos mananciais (Barreto Filho et al., 2000).

Segundo Boman (1989), a uniformidade é um indicador da igualdade (ou desigualdade) das taxas de aplicação da água. Uniformidade alta é importante para irrigação em terras arenosas, onde a redistribuição lateral da água é limitada. Excessos de aplicação de água nesses solos resultam freqüentemente em lâmina percolada e lixiviação de nutrientes para fora da zona radicular. A uniformidade elevada pode ser especialmente desejável para fertirrigação ou quimigação, visto que as aplicações de nutrientes ou outros produtos químicos não serão mais uniformes do que a distribuição de água.

Em agricultura irrigada, os parâmetros envolvidos, sejam relativos à água, ao solo, à planta ou à atmosfera, exibem um comportamento variável no espaço e no tempo, requerendo uma boa análise estatística para interpretação dos dados. Uma análise exploratória adequada dos dados constitui o ponto de partida para observar o comportamento das variáveis de interesse, o que pode ser feito por meio de técnicas estatísticas descritivas gerais e espaciais. Essa análise servirá de base para orientar na decisão de se assumir algum tipo de correlação espacial dos dados, o que é de fundamental importância nas análises geoestatísticas (Queiroz et al., 1997).

Ante o exposto, o objetivo deste trabalho foi avaliar a variabilidade espacial da vazão e pressão em uma subunidade de irrigação por microaspersão com emissores novos e usados, empregando técnicas da estatística clássica e da geoestatística na área irrigada.

\section{MATERIAL E MÉTODOS}

O trabalho foi realizado numa área cultivada com graviola (Annona muricata L.), do perímetro irrigado de São Gonçalo, no município de Sousa, PB, cujo clima é do tipo BSh, semi-árido quente. A temperatura média anual é de $27,8^{\circ} \mathrm{C}$, com precipitação média anual de $894 \mathrm{~mm}$, concentrada nos meses de janeiro a maio. A umidade relativa média do ar é de $58 \%$, e a velocidade média do vento é de $2,5 \mathrm{~m} \mathrm{~s}^{-1}$.

Num sistema de irrigação por microaspersão, instalado há dois anos com a cultura da graviola, com um espaçamento de $6,0 \times 5,0 \mathrm{~m}$, utilizando-se um microaspersor por planta, avaliou-se a subunidade com linha terciária em PVC de $144 \mathrm{~m}$ na qual eram conectadas as laterais de polietileno com $30 \mathrm{~m}$, possuindo 7 microaspersores espaçados em $5 \mathrm{~m}$ numa área irrigada de $4320 \mathrm{~m}^{2}$. O microaspersor autocompensante usado era da marca EINDOR 86, e de acordo com as especificações do fabricante, possuía uma vazão de $50 \mathrm{~L} \mathrm{~h}^{-1}$ a uma pressão de serviço entre 150 a $200 \mathrm{kPa}$. Foram escolhidos 28 microaspersores, dentro da subunidade de irrigação, sendo espaçados na direção da tubulação lateral em 10 m e na direção da terciária em 18 m (Figura 1), onde se determinou a vazão e a pressão de cada emissor em campo, utilizando para isso uma proveta de $1000 \mathrm{~mL}$, como coletor da água, um cronômetro e um manômetro digital. Durante um período de 2 horas, o sistema de irrigação era mantido ligado, e a coleta d'água era feita por um tempo de $30 \mathrm{~s}$, obtendo-se médias de três repetições para cada ponto. Após a coleta dos dados com os microaspersores usados, permutou-se por novos, realizando-se outro ensaio. 


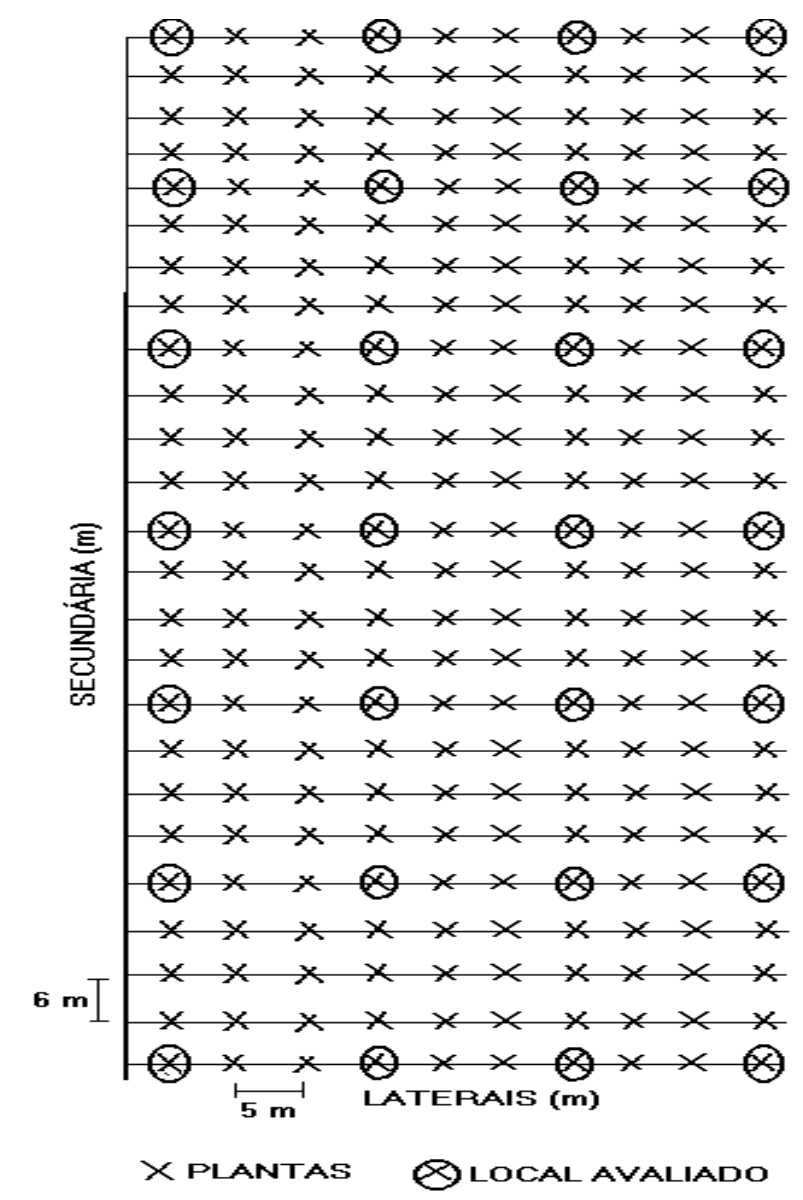

Figura 1. Esquema dos pontos de coleta de dados na subunidade de irrigação.

A partir dos resultados obtidos na subunidade, determinou-se a uniformidade de emissão, segundo as metodologias de Merrian e Keller (1978) e ASAE (1996). Determinou-se a variação de pressão e vazão ao longo das linhas laterais e das secundárias, utilizando-se as seguintes equações:

$$
\begin{aligned}
& \Delta Q=\frac{\text { Qmáx }- \text { Qmín }}{\text { Qmáx }} * 100 \\
& \Delta P=\frac{\text { Pmáx }- \text { Pmín }}{\text { Pmáx }} * 100
\end{aligned}
$$

em que:

$\Delta \mathrm{P} \quad$ - variação de pressão, $\%$

Pmáx - valor máximo de pressão, $\mathrm{kPa}$

Pmín - valor mínimo de pressão, $\mathrm{kPa}$

$\Delta \mathrm{Q}$ - variação de vazão na lateral, \%

Qmáx - valor máximo de vazão, $\mathrm{L} \mathrm{h}^{-1}$

Qmín - valor mínimo de vazão, $\mathrm{L} \mathrm{h}^{-1}$ 
A partir dos valores de vazão e de pressão encontrados na subunidade, determinou-se a uniformidade de irrigação, segundo as metodologias:

\subsection{Metodologia de Merrian e Keller (1978)}

$$
C U=100\left(\frac{q_{25 \%}}{q_{\text {med }}}\right)
$$

em que:

CU - coeficiente de uniformidade de irrigação da subunidade avaliada em campo, \%

$\mathrm{q}_{25 \%}$ - média de $25 \%$ do total de microaspersores com as menores vazões $\left(\mathrm{L} \mathrm{h}^{-1}\right)$

$\mathrm{q}_{\text {med }}$ - média das vazões coletadas nos microaspersores na subunidade $\left(\mathrm{L} \mathrm{h}^{-1}\right)$

$$
C U p=100 *\left(\frac{P_{25 \%}}{P M}\right)^{X}
$$

em que:

CUp - coeficiente de uniformidade de pressão, \%

$\mathrm{PM}$ - pressão média da subunidade $(\mathrm{kPa})$

$\mathrm{P}_{25 \%}$ - pressão média de $25 \%$ das pressões mais baixas dentro da subunidade avaliada, $\mathrm{kPa}$

$\mathrm{x}$ - expoente de descarga do emissor, calculado pela Eq. 5:

$$
x=\frac{\log \left(\bar{q}_{1} / \bar{q}_{2}\right)}{\log \left(h_{1} / h_{2}\right)}
$$

em que:

$\mathrm{q}_{1}$ - média da vazão de quatro emissores obtida com a menor pressão, $\mathrm{h}_{1}$

$\mathrm{q}_{2}$ - média da vazão de quatro emissores obtida com a maior pressão, $\mathrm{h}_{2}$

O fator de correção das vazões foi determinado mediante a seguinte equação:

$$
F C V=\left(\frac{P_{25 \%}}{P M_{\text {mín }}}\right)^{X}
$$

em que:

FCV - fator de correção das vazões

$\mathrm{PM}_{\text {min }}$ - média de todas as pressões mínimas da subunidade, $\mathrm{kPa}$

$\mathrm{P}_{25 \%}$ - pressão média de $25 \%$ das pressões mais baixas da subunidade, $\mathrm{kPa}$

$\mathrm{x}$ - expoente de descarga do emissor

A uniformidade de irrigação do sistema (CUS) foi dada pela Eq. 7:

$$
C U S=C U^{*} F C V
$$

A eficiência de aplicação (EA) sob irrigação completa, segundo Merrian e Keller (1978), foi estimada por:

$$
E A=0,9 * C U
$$




\subsection{Metodologia da ASAE (1996)}

A avaliação da uniformidade de irrigação, segundo a ASAE (1996), é baseada no método de estimação de uniformidade em campo, proposto por Bralts e Kesner (1983), fundamentado no coeficiente de uniformidade estatístico. Selecionou-se uma subunidade de irrigação que estava funcionando em concordância com as condições normais de trabalho e avaliou-se a uniformidade de aplicação de água dentro da subunidade a partir da Eq. 9:

$$
C U E=(1-C V T) * 100
$$

em que:

CUE - coeficiente de uniformidade estatístico de irrigação

CVT - coeficiente de variação total de vazão na subunidade

Para a determinação do coeficiente de variação de vazão total (CVT) para a subunidade, foi usada a seguinte equação:

$$
C V T=\frac{S_{q}}{q_{\text {med }}}
$$

em que:

$\mathrm{S}_{\mathrm{q}} \quad$ - desvio padrão da vazão dos emissores $\left(\mathrm{L} \mathrm{h}^{-1}\right)$

$\mathrm{q}_{\text {med }}$ - vazão média dos emissores na subunidade $\left(\mathrm{L} \mathrm{h}^{-1}\right)$

Com as pressões medidas na subunidade, obteve-se um coeficiente de variação da vazão por causas hidráulicas $(\mathrm{CVH})$, pela expressão:

$$
C V H=\frac{S_{h}}{h_{\text {med }}}
$$

em que:

$\mathrm{S}_{\mathrm{h}}$ - desvio padrão das pressões medidas $(\mathrm{kPa})$

$\mathrm{h}_{\text {med }}$ - pressão média dos emissores na subunidade $(\mathrm{kPa})$

O coeficiente da variação do desempenho dos emissores (CVE) foi dado por:

$$
C V E=\sqrt{C V T^{2}-x^{2} * C V H^{2}}
$$

Por meio da estatística descritiva determinaram-se medidas de posição: média aritmética e a mediana amostral e as medidas de dispersão, variância, desvio-padrão amostral e coeficiente de variação. Esse procedimento foi importante para o conhecimento preliminar da distribuição da vazão e pressão no sistema de irrigação. Com um total de 28 pontos amostrais do conteúdo de água no solo, analisaram-se a estrutura e a dependência espacial, pela análise geoestatística e pelos semivariogramas experimentais (Andrade, 2002), em que a dependência espacial é definida pelo alcance (Ao), o erro cometido devido à distância de amostragem é definido pelo efeito pepita (Co), o ponto onde toda semivariância da amostra dos dados é de influência aleatória é medido pela variância estrutural ou espacial $(\mathrm{Cl})$, e o patamar $(\mathbf{C})$ que corresponde, aproximadamente, ao valor da variância total da variável em estudo, é obtido por $\mathrm{C}=(\mathrm{Co}+\mathrm{Cl})$. A dependência espacial entre as observações pode ser expressa pelo semivariograma, estimado pela seguinte expressão. 


$$
\bar{y}(h)=\frac{1}{2 N(h)} \sum_{i=1}^{N(h)}\left[z\left(x_{i}\right)-z\left(x_{i}+h\right)\right]^{2}
$$

em que:

$\bar{y}(h)$ - valor da semivariância estimado para a distância de separação (h) entre as medidas.

$\mathrm{Z}\left(\mathrm{x}_{\mathrm{i}}\right)$ - valor da variável para a posição xi não estimado (verdadeiro).

$\mathrm{Z}\left(\mathrm{x}_{\mathrm{i}}+\mathrm{h}\right)$ - valor da mesma variável na posição $\mathrm{x}_{\mathrm{i}}+\mathrm{h}$ em qualquer direção.

$\mathrm{h}$ - distância de separação entre as medidas.

$\mathrm{N}(\mathrm{h})$ - número de pares experimentais de dados medidos de $\mathrm{Z}\left(\mathrm{x}_{\mathrm{i}}\right)$ e $\mathrm{Z}\left(\mathrm{x}_{\mathrm{i}}+\mathrm{h}\right)$.

O gráfico de $\bar{y}(h)$ versus os valores correspondentes de h é chamado semivariograma, sendo, então, uma função do vetor $\mathrm{h}$, e, portanto depende tanto de sua magnitude como da direção de h. Modelos matemáticos devem ser ajustados aos semivariogramas, os quais permitem visualizar a natureza da variação espacial da vazão e pressão no sistema de irrigação, além de serem necessários para outras aplicações, como, por exemplo, krigagem. Para se analisar o grau de dependência espacial das variáveis em estudo, utilizou-se à classificação de Cambardella et al. (1994) em que são considerados de dependência espacial forte os semivariogramas que têm efeito pepita $\leq 25 \%$ do patamar, de dependência espacial moderada quando o efeito pepita está entre 25 a $75 \%$ e de dependência espacial fraca, quando o efeito pepita é $>75 \%$.

Foi construído o semivariograma experimental e definido o modelo de ajuste, de modo que a curva que melhor se ajustou aos pontos obtidos representa a magnitude, alcance e a intensidade da variabilidade espacial da vazão e pressão no sistema de irrigação. A decisão pelo melhor modelo e respectivos coeficientes foi tomada com base na validação cruzada.

Existindo a dependência espacial, as estimativas para pontos não observados são obtidas pela técnica da krigagem. Essa é uma técnica de interpolação não tendenciosa, que possui variância mínima, sendo cada estimativa obtida pelo cálculo de uma média ponderada de um conjunto de observações ao redor de uma vizinhança (Andrade, 2002) em que o valor de $\bar{Z}\left(x_{0}\right)$, associado à posição $\mathrm{x}_{0}$, pode ser obtido pela expressão.

$$
\bar{Z}\left(x_{0}\right)=\sum_{i=1}^{N} \lambda_{i} Z\left(x_{i}\right)
$$

Sendo: $\bar{Z}\left(x_{0}\right)$ valores estimados da variável em estudo, $\mathrm{x}_{0}$ o ponto a ser estimado, $\mathrm{N}$ o número de vizinhos utilizado na estimativa $\bar{Z}\left(x_{0}\right), \lambda_{\mathrm{i} \text { os }}$ pesos ponderados associados a cada valor medido de $\bar{Z}\left(x_{0}\right)$.

Por meio de técnicas de interpolação da krigagem foram construídos os mapas de isolinhas e de superfície em três dimensões (3D) representativos da distribuição espacial da vazão e pressão nos emissores, com o auxilio do programa Surfer 7.0 (Golden Software, 2001) e dos parâmetros de cada modelo de semivariogramas ajustados aos dados de vazão e pressão nos emissores, utilizando-se o software GS+ "Geoestatístics for Environmental Sciences" (GS+, 2000). 


\section{RESULTADOS E DISCUSSÃO}

De acordo com os resultados da avaliação de campo (Tabela 1), a subunidade com microaspersores usados apresentou uma baixa uniformidade de distribuição de água e, conseqüentemente, uma baixa eficiência de irrigação, devido à baixa pressão observada no sistema, provocando uma grande variação de vazão nos emissores ao longo das linhas laterais. $\mathrm{Na}$ permuta de emissores usados por novos, foi possível observar que o valor de pressão média aumentou, passando de 112 para $122 \mathrm{kPa}$, proporcionando também um aumento de vazão de 32,9 para $36,9 \mathrm{~L} \mathrm{~h}^{-1}$, mesmo assim permanecendo baixos os valores de pressão e vazão da subunidade. As variações de pressão e vazão na subunidade foram de 28,24 e $36,36 \%$, respectivamente, sendo inferiores as variações com microaspersores novos, porém elevadas comparando-se com valores apresentados por Keller e Karmeli (1974), que recomendam uma variação máxima de pressão de $20 \%$ e de vazão de $10 \%$ na subunidade.

Tabela 1. Valores das variáveis avaliadas na subunidade de irrigação em condições reais de campo, para microaspersores novos e usados.

\begin{tabular}{lrr}
\hline \multirow{2}{*}{ Variáveis } & \multicolumn{2}{c}{ Emissores } \\
\cline { 2 - 3 } & Usados & Novos \\
\hline Vazão Média $\left(\mathrm{L} \mathrm{h}^{-1}\right)$ & 32,9 & 36,9 \\
Vazão Máxima $\left(\mathrm{L} \mathrm{h}^{-1}\right)$ & 40 & 47 \\
Vazão Mínima $\left(\mathrm{L} \mathrm{h}^{-1}\right)$ & 25 & 26 \\
Vazão 25\% Mínima $\left(\mathrm{L} \mathrm{h}^{-1}\right)$ & 27,9 & 35,6 \\
Desvio Padrão de vazão $\left(\mathrm{L} \mathrm{h}^{-1}\right)$ & 3,7 & 4,44 \\
Variação de Vazão (\%) & 36,36 & 43,59 \\
Pressão Média (kPa) & 112 & 122 \\
Pressão Máxima (kPa) & 131 & 148 \\
Pressão Mínima (kPa) & 94 & 96 \\
Pressão 25\% Mínima (kPa) & 100 & 107 \\
Desvio Padrão de Pressão (kPa) & 9,8 & 12,4 \\
Variação de Pressão (\%) & 28,24 & 35,14 \\
Expoente de Descarga do Emissor - x & 0,87 & 0,64 \\
Coefic. de Unifor. de Irrigação (\%) - CU & 84,69 & 96,5 \\
Coefic. de Unifor. de Pressão (\%) - CUP & 89,59 & 87,8 \\
Fator de Correção das Vazões - FCV & 1,05 & 1,07 \\
Coefic. de Unifor. do Sistema (\%) - CUS & 89,25 & 100 \\
Eficiência de Aplicação (\%) - EA & 76,22 & 86,86 \\
Coefic. de Variação Total de Vazão (\%) - CVT & 0,11 & 0,12 \\
Coefic. de Unifor. Estatístico. (\%) - CUE & 88,82 & 88,0 \\
Coefic. de Variação da Vazão (\%) - CVH & 0,09 & 0,10 \\
Coefic. de Variação Estat. da Vazão (\%) - CVE & 0,01 & 0,01 \\
\hline
\end{tabular}

O coeficiente de variação da vazão $(\mathrm{CVH})$ e o coeficiente de variação total de vazão (CVT), respectivamente, de 0,10 e 0,12 foram altos, no entanto, sem diferença em relação aos valores com os microaspersores usados, próximo do encontrado por Zanini et al. (1998) que avaliando um sistema de irrigação por microaspersão, encontraram coeficientes de variação de vazão total variando de 0,85 a 0,11 . O coeficiente de variação estatístico da vazão (CVE) de 0,01 , que indica a variação da vazão devido a causas hidráulicas, não variou entre as duas avaliações realizadas, e foi considerado baixo, caracterizando como bom o coeficiente de 
variação de fabricação dos emissores analisados, indicando que parte da variação da vazão foi devido a diferentes causas como: obstrução do emissor, tempo de uso e da sustentabilidade de variação da vazão com o tempo; no entanto, pode-se dizer que, na subunidade avaliada com microaspersores novos, a variação de vazão foi devida, principalmente à variação de pressão.

O coeficiente de uniformidade de irrigação (CU) de 84,69\%, classificado como bom, foi para $96,5 \%$ com a troca dos microaspersores passando assim para excelente; para o coeficiente de uniformidade estatístico (CUE) não houve diferença considerável com a troca, o qual baixou de 88,82 para $88,0 \%$, permanecendo como bom, de acordo com a ASAE (1996).

O coeficiente de variação de pressão $\left(\mathrm{CU}_{\mathrm{P}}\right)$ de $87,8 \%$ baixou em relação à avaliação com os microaspersores usados, mas ainda não chegando a um valor satisfatório, de acordo com Keller e Bliesner (1990), que recomendam valores mínimos em torno de $80 \%$ como valor satisfatório. A eficiência de aplicação (EA) teve um aumento considerável de 10,64\% com a troca dos microaspersores, constatando-se que houve um ganho de eficiência na aplicação de água com os microaspersores novos, possivelmente devido às mudanças da área de passagem de fluxo, causando uma melhor uniformidade.

O expoente de descarga (x) dos emissores usados de 0,87 baixou para 0,64 com a troca por novos, verificando-se, então, que houve perda de qualidade com o tempo de uso, causando mais variação de vazão do que de pressão.

Os dados dos principais parâmetros estatísticos da vazão e da pressão da subunidade são apresentados na Tabela 2. Os resultados mostram valores máximo e mínimo de vazão de 40 e $25 \mathrm{~L} \mathrm{~h}^{-1}$ dos 28 microaspersores com três anos de uso, avaliados em condições reais de uso, com coeficiente de variação de $11,39 \%$. A vazão média foi de $32,93 \mathrm{~L} \mathrm{~h}^{-1}$, sendo menor que a média do valor obtido em laboratório de $39,39 \mathrm{~L} \mathrm{~h}^{-1}$ com o microaspersor submetido a uma pressão de $150 \mathrm{kPa}$. Quando se trocou os microaspersores usados por novos, o valor de vazão máxima foi de 46,80 $\mathrm{L} \mathrm{h}^{-1}$, havendo um aumento de $3,93 \mathrm{~L} \mathrm{~h}^{-1}$ na vazão média e um coeficiente de variação de $12,05 \%$.

Tabela 2. Valores dos parâmetros estatísticos e teste de KomolgorovSminov (KS), para a vazão e pressão de microaspersores usados e novos na subunidade de irrigação.

\begin{tabular}{lcccc}
\hline \multirow{2}{*}{ Parâmetros } & \multicolumn{2}{c}{ Vazão $\left(\mathbf{L ~ h ~}^{\mathbf{- 1}}\right)$} & \multicolumn{2}{c}{ Pressão (kPa) } \\
\cline { 2 - 5 } & Usado & Novo & Usado & Novo \\
\hline Média & 32,93 & 36,86 & 112 & 122 \\
Mediana & 34,00 & 36,00 & 115 & 122 \\
Desvio Padrão & 3,7 & 4,44 & 10 & 12 \\
Coeficiente de & 11,39 & 12,05 & 8,76 & 10,14 \\
Variação & 14,07 & 19,72 & 95,84 & 154,02 \\
Variância & 25,00 & 26,40 & 94 & 96 \\
Mínimo & 40,00 & 46,80 & 131 & 148 \\
Máximo & $0,257^{* *}$ & $0,218^{* *}$ & $0,133^{* *}$ & $0,159^{* *}$ \\
\hline Teste KS & & \multicolumn{3}{c}{}
\end{tabular}

** significativo a $1 \%$ de probabilidade.

Para os valores de pressão na subunidade, foi constatado que, para os microaspersores usados, a máxima pressão medida foi de $131 \mathrm{kPa}$, com um valor médio de $112 \mathrm{kPa}$; realizada a troca pelos microaspersores novos, a pressão teve um aumento médio de $10 \mathrm{kPa}$. 
A uniformidade da irrigação de um sistema de irrigação localizada pode ser melhorada se a redistribuição de água no solo for considerada. Para tanto é necessário descrever a distribuição espacial do armazenamento de água no solo, uma vez que mesmo em um solo considerado homogêneo, a variabilidade das propriedades físico-hídricas pode ser grande. Hamlett et al. (1986) afirmam que análises espaciais de dados têm sido cada vez mais empregadas, sendo baseadas na geoestatística, a qual considera as características estruturais e aleatórias de uma variável espacialmente distribuída.

São apresentados na Tabela 3 os valores dos parâmetros dos semivariogramas analisados de vazão e pressão da subunidade com microaspersores novos e usados, em que se pode verificar que o modelo matemático que melhor se ajustou à vazão dos microaspersores novos e usados, foi, respectivamente, o exponencial e esférico; para a pressão o modelo foi o inverso da vazão, ou seja, esférico para os novos e exponencial para os usados. Observa-se que quando o modelo foi esférico, ocorreram as menores variações não explicadas (efeito pepita) na vazão e na pressão; também é possível verificar que o alcance aumentou com o modelo esférico para a vazão e pressão. Pelos valores do patamar se constata que com a troca dos microaspersores os valores de vazão teriam de aumentar para se conseguir uma estabilidade.

Tabela 3. Estimativa dos parâmetros dos modelos ajustados aos semivariogramas para os valores de vazão e pressão na subunidade com microaspersores novos e usados.

\begin{tabular}{|c|c|c|c|c|c|c|}
\hline \multicolumn{2}{|c|}{ Parâmetros } & \multirow{2}{*}{$\begin{array}{r}\text { Efeito } \\
\text { Pepita } \\
\text { Co }\end{array}$} & \multirow{2}{*}{$\begin{array}{r}\text { Patamar } \\
\mathrm{Co}+\mathrm{Cl}\end{array}$} & \multirow{2}{*}{$\begin{array}{r}\begin{array}{c}\text { Alcance } \\
\text { (m) }\end{array} \\
\text { Ao }\end{array}$} & \multirow{2}{*}{$\begin{array}{c}\begin{array}{c}\text { Dependência } \\
\text { Espacial (\%) }\end{array} \\
(\mathrm{Co} /(\mathrm{Co}+\mathrm{Cl})) \\
100\end{array}$} & \multirow{2}{*}{$\begin{array}{l}\text { Aleatoriedade } \\
E=(\mathrm{Co} / \mathrm{Cl})\end{array}$} \\
\hline Variáveis & Modelo & & & & & \\
\hline $\begin{array}{l}\text { Vazão - } \\
\text { Novo }\end{array}$ & Exponencial & 5 & 35 & 212 & $14,2 \mathrm{ft}$ & $0,16 \mathrm{~s}$ \\
\hline $\begin{array}{l}\text { Vazão - } \\
\text { Usado }\end{array}$ & Esférico & 1 & 33 & 218 & $3,0 \mathrm{ft}$ & $0,031 \mathrm{~s}$ \\
\hline $\begin{array}{l}\text { Pressão - } \\
\text { Novo }\end{array}$ & Esférico & 12 & 334 & 225 & $3,6 \mathrm{ft}$ & $0,54 \mathrm{~ms}$ \\
\hline $\begin{array}{l}\text { Pressão - } \\
\text { Usado }\end{array}$ & Exponencial & 43 & 137 & 58 & $31,38 \mathrm{ft}$ & $0,45 \mathrm{~ms}$ \\
\hline
\end{tabular}

$\mathrm{ft}$ - forte; $\mathrm{s}$ - significativo; $\mathrm{ms}$ - muito significativo.

A Figura 2 apresenta o semivariograma da vazão e da pressão dos microaspersores usados e novos. Para o semivariograma da vazão com microaspersores usados (Figura 2A), o modelo foi esférico com uma variância de vazão em função da distância dos emissores, há três anos no campo, que apresenta efeito pepita de $1 \mathrm{~L} \mathrm{~h}^{-1}$, valor menor se comparado com os novos (Figura 2B), patamar de $33 \mathrm{~L} \mathrm{~h}^{-1}$, alcance de $218 \mathrm{~m}$, com uma dependência espacial menor de 3\%, classificada como forte de acordo com a classificação de Cambardella et al. (1994), e um grau de aleatoriedade significativo de 0,031. Para o semivariograma da vazão para os emissores novos (Figura 2B), o modelo foi exponencial, exibindo um efeito pepita de $5 \mathrm{~L} \mathrm{~h}^{-1}$, um patamar de $35 \mathrm{~L} \mathrm{~h}^{-1}$ e um alcance em que existe uma influência de uma amostra sobre a outra de $212 \mathrm{~m}$. A dependência espacial entre os emissores novos foi de $14,28 \%$ considerada como forte, de acordo com a classificação de Cambardella et al. (1994), sendo essa forte dependência justificada por se tratar de um sistema que está interligado; no entanto, 
os valores de vazão dependem do estado do emissor como mostra a aleatoriedade muito significativa de 0,16 entre esses valores.

A.

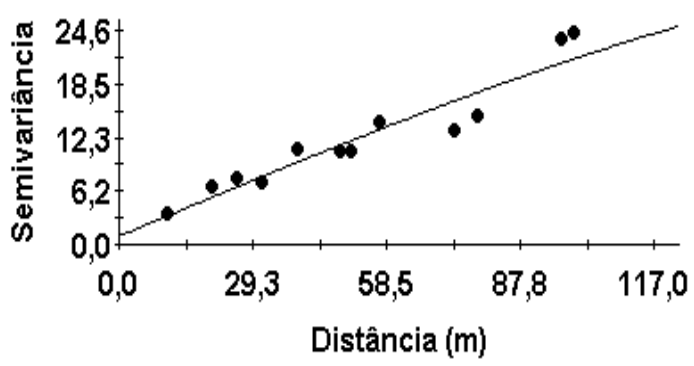

C.

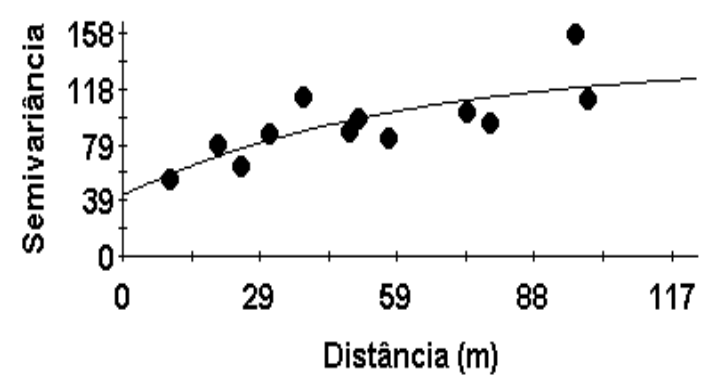

B.

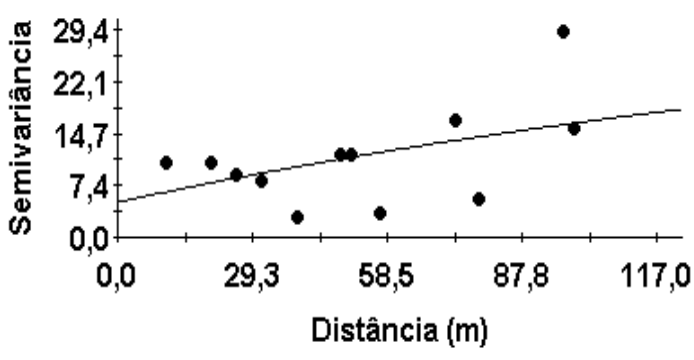

D.

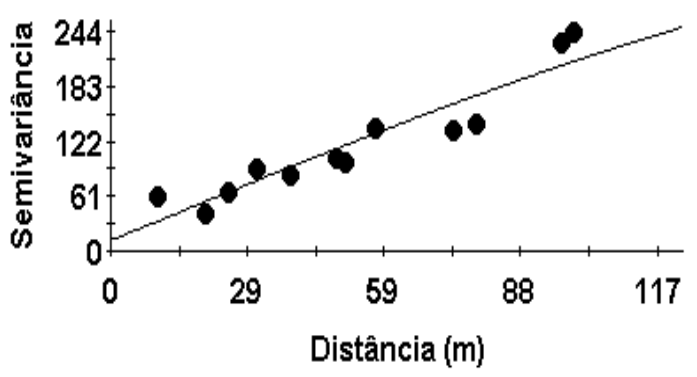

Figu999ra 2. Semivariogramas de vazão, para microaspersores usados (A) e novos (B), e de pressão, para microaspersores usados $(\mathrm{C})$ e novos (D), na subunidade de irrigação, mostrando o modelo ajustado aos dados em função da distância.

No semivariograma da pressão para os microaspersores usados (Figura 2C), o modelo foi exponencial, com um efeito pepita também baixo de $43 \mathrm{kPa}$, e o patamar de $137 \mathrm{kPa}$, expressando que a estabilidade seria atingida com essa pressão, sendo esperado esse resultado, pois com os emissores novos a pressão do sistema aumentou. $\mathrm{O}$ alcance foi de 58 $\mathrm{m}$, mostrando uma dependência espacial forte de 31,38\% e um grau de aleatoriedade muito significativo de 0,45 . O semivariograma da pressão com os microaspersores novos (Figura 2D), apresenta um modelo esférico, com um efeito pepita muito baixo de $12 \mathrm{kPa}$, patamar igual a $334 \mathrm{kPa}$ e um alcance de $225 \mathrm{~m}$, observando-se uma dependência espacial de 3,6\%, classificada novamente como forte, e um grau de aleatoriedade de 0,037 classificado como pouco significativo.

A Figura 3 exibe os mapas de isolinhas da vazão e da pressão, sendo a coordenada $\mathrm{x}$ a direção da linha de derivação e a coordenada y a direção das laterais. Observa-se que para os microaspersores usados (Figura 3A) não ocorreu muita variação na vazão, com uma média de $32,93 \mathrm{~L} \mathrm{~h}^{-1}$, ocorrendo no início da área, vazões maiores nos pontos de emissão e valores mínimos de $25 \mathrm{~L} \mathrm{~h}^{-1}$ no final. Já com os microaspersores trocados por novos (Figura 3B), a média aumentou para $36,86 \mathrm{~L} \mathrm{~h}^{-1}$, chegando a $46 \mathrm{~L} \mathrm{~h}^{-1}$ no início em que a vazão foi maior, diminuindo em direção ao final da tubulação secundária, com um valor mínimo de $26,40 \mathrm{~L} \mathrm{~h}^{-}$ 1. Observa-se que entre as causas dessa diminuição de vazão, uma delas foi a pressão do 
sistema, que quando comparado com os mesmos microaspersores testados em laboratório a uma pressão de $150 \mathrm{kPa}$, em que o coeficiente de variação de fabricação foi de apenas $0,01 \%$, a vazão média foi de $39,39 \mathrm{~L} \mathrm{~h}^{-1}$.

A.

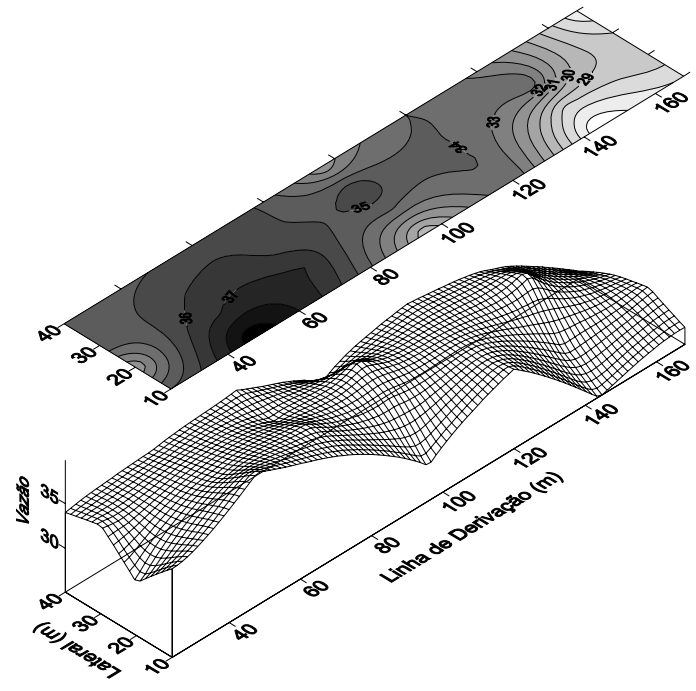

C.
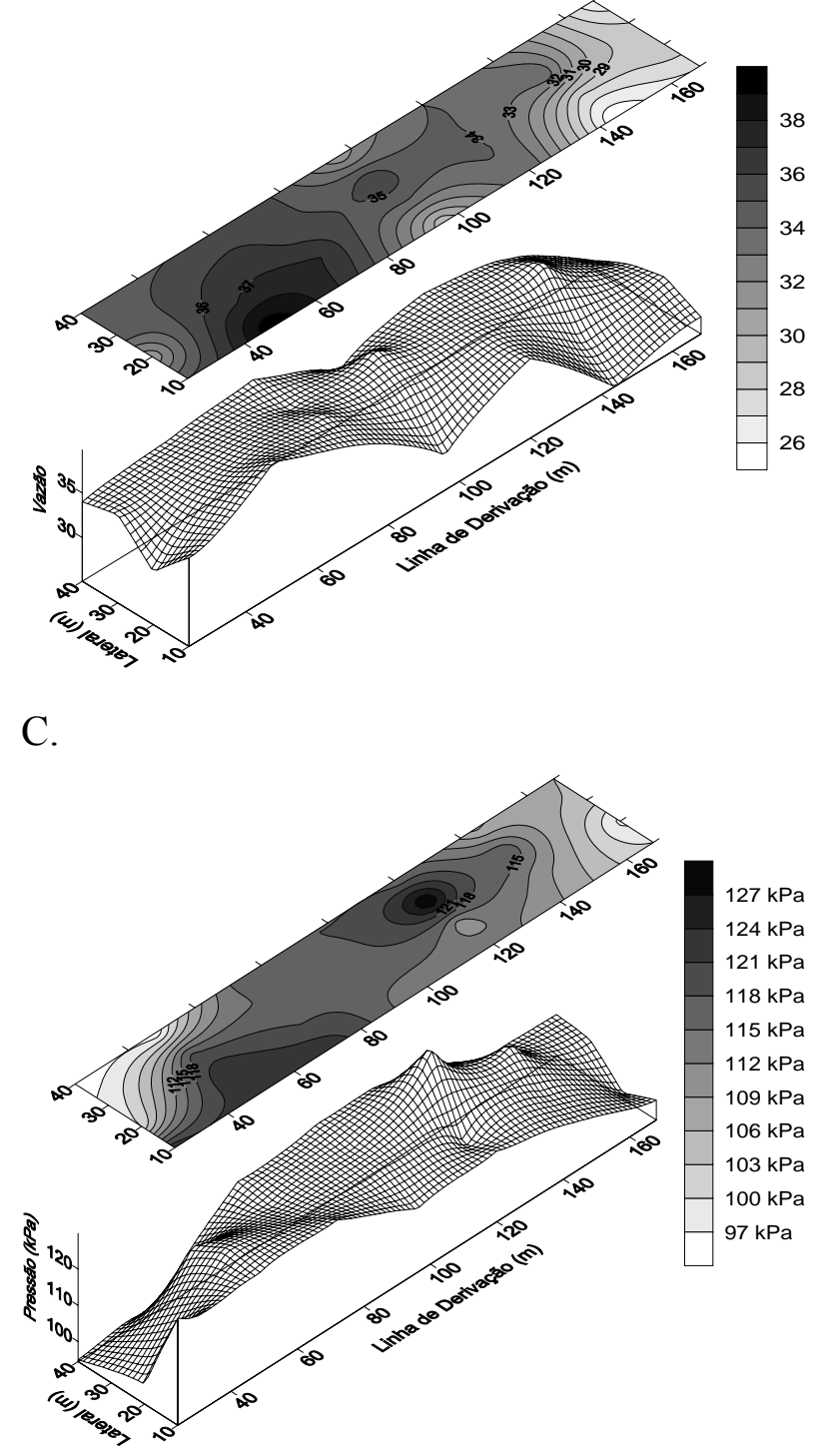

B.

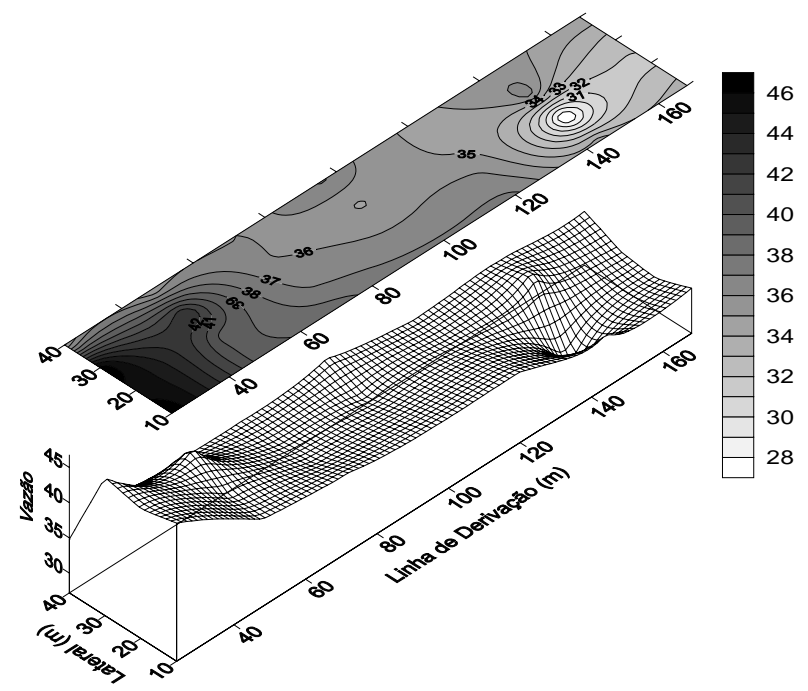

D.

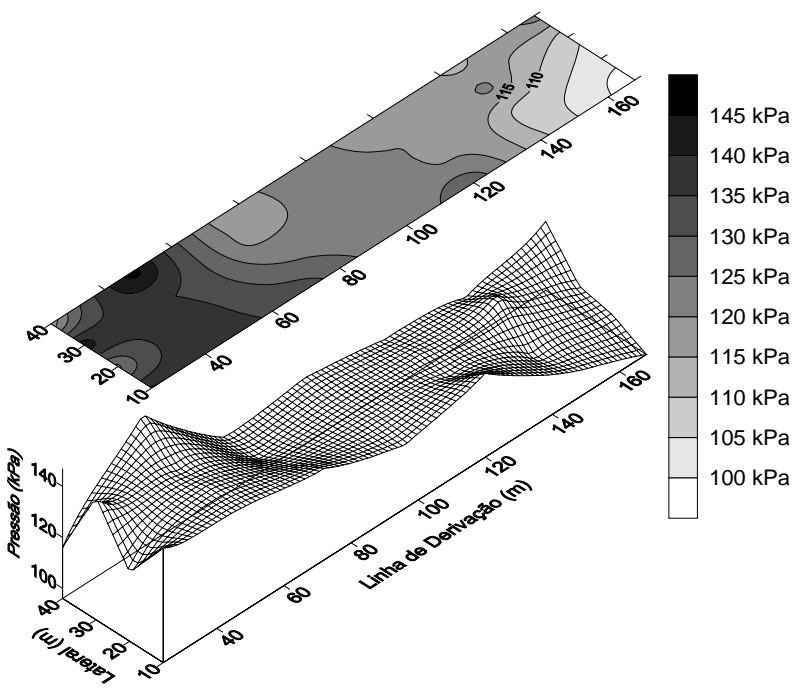

Figura 3. Mapas de isolinhas da vazão, para microaspersores novos (A) e usados (B) e da pressão, para microaspersores usados (C) e novos (D) na subunidade de irrigação.

O mapa de isolinhas da pressão para microaspersores usados (Figura 3C), gerado a partir de um modelo exponencial, apresenta valores mínimo e máximo de 94 e $131 \mathrm{kPa}$, respectivamente, em que é possível observar que os maiores valores estão no início da malha instalada na subunidade. Com as medidas de pressão nos pontos de emissão, tendo os microaspersores sido trocados por novos, pode-se observar pelo mapa de isolinhas (Figura 3D), que a média de $122 \mathrm{kPa}$ foi maior que na avaliação anterior, ou seja, para os microaspersores usados, mas apresentando uma variação de pressão maior de $52 \mathrm{kPa}$ entre os valores máximo e mínimo. 


\section{CONCLUSÕES}

Quando se trocaram em campo os emissores usados por novos, o coeficiente de uniformidade de irrigação, o coeficiente de uniformidade do sistema e a eficiência de aplicação d'água aumentaram em 11,9, 10,58 e 10,75\%, respectivamente.

A distância máxima (alcance) onde os dados estimados de vazão e pressão se correlacionam espacialmente extrapolou o comprimento da linha de derivação para a vazão com microaspersores novos e usados e para a pressão só com microaspersores novos.

O efeito pepita para pressão no sistema foi menor quando se trocaram os microaspersores usados por novos, ocorrendo o inverso para a vazão.

Houve um aumento tanto da pressão como da vazão quando se trocaram os microaspersores usados por novos.

\section{AGRADECIMENTOS}

Os autores agradecem ao Conselho Nacional de Desenvolvimento Científico e Tecnológico, CNPq, pelo suporte financeiro, fundamental ao desenvolvimento desta pesquisa.

\section{REFERÊNCIAS}

ANDRADE A. R. S. de; Aplicação da teoria fractal e da geoestatística na estimativa da condutividade hidráulica saturada e do espaçamento entre drenos em solos de vázea.. 2002. 181f. Tese (Doutorado em Irrigação e Drenagem) - UNESP, Botucatu, 2002.

ABREU, J. M. H.; LOPEZ, J. R.; REGALADO, A. P.; HERNANDEZ, J. F. G. El riego localizado. Madrid: Instituto Nacional de Investigaciones Agrarias, 1987. 317p.

AMERICAN SOCIETY OF AGRICULTURAL ENGINEERS - ASAE. Field evaluation of micro irrigation systems. EP458. St. Joseph: ASAE, 1996. p.792-797.

BARRETO FILHO, A. A.; DANTAS NETO, J.; MATOS, J. A.; GOMES, E. M. Desempenho de um sistema de irrigação por microaspersão, instalado em campo, Revista Brasileira de Engenharia agrícola e ambiental, Campina Grande, v. 4, n. 3, p. 309-314, 2000.

BOMAN, B. J. Distribution patterns of micro irrigations spinner and spray emitters. Applied Engineering Agriculture, St. Joseph, v. 5, n. 2, p. 50-60, 1989.

BRALTS, V. F.; KESNER, C. Drip irrigation field uniformity estimation. Transactions of the ASAE, St. Joseph, v. 24, n. 5, p. 1369-1374, 1983.

CAMBARDELLA, C. A.; MOORMAN, T. B.; NOVACK, J. M.; PARKIN, T. B.; KARLEN, D. L.; TURCO R. F.; KNOPKA, A. E. Field-scale variability of soil proprieties in central Iowa soil. Soil Science, v. 58, p. 1240-1248, 1994.

G+: Geostatistics for Environmental Science. Version 5.0. Michigan: Gamma Design Software, 2000.

GOLDEN SOFTWARE: surfer for Windows. Surface mapping system. New York, Version 7, 2001. 
HAMLETT, J. M.; HORTON, R.; CRESSIE, N. A. C. Resistant and exploratory techniques for use in semivariogram analyses. Soil Science Society of America Journal, v. 50 p. 868-875, 1986.

KELLER, J.; BLIESNER, R. D. Sprinkler and trickle irrigation. New York: Van Nostrand Reinhold, 1990. 652p.

KELLER, J.; KARMELI, D. Trickle irrigation design parameters. Transactions of the ASAE, St. Joseph, v. 17, n. 4, p. 678-684, 1974.

MERRIAN, J. L.; KELLER, J. Farm irrigation system evaluation: a guide for management. Logan: Utah State University, Agricultural and Irrigation Engineering Department, 1978. 271p.

QUEIROZ, J. E.; GONÇALVES, A. C.; SOUTO, J. S.; FOLEGATTI, M. V. Avaliação e monitoramento da salinidade do solo. In: GHEIY, H. R.; QUEIROZ, J. E.; MEDEIROS, J. F. Manejo e controle da salinidade na agricultura irrigada. Campina Grande: UFPB/SBEA, p. 69-111, 1997. Cap. 3.

ZANINI, J. R.; PAVANI, L. C.; TAROZZO, M. Avaliação da vazão de um sistema de irrigação por microaspersão em cultura de bananeira. In: CONGRESSO BRASILEIRO DE ENGENHARIA AGRÍCOLA, 27, 1998, Poços de Caldas. Resumos... Poços de Caldas: SBEA, 1998, v. 2, p.290-292. 